\title{
Ball Classification through Object Detection using Deep Learning for Handball
}

\author{
Arzielah Ashiqin Alwi ${ }^{1}$, Ahmad Najmuddin Ibrahim¹, Muhammad Nur Aiman Shapiee ${ }^{1}$, Muhammad Ar Rahim Ibrahim¹, Mohd Azraai \\ Mohd Razman ${ }^{1 *}$ and Ismail Mohd Khairuddin ${ }^{*}$ \\ ${ }^{1}$ Faculty of Manufacturing and Manufacturing Engineering Technology, Universiti Malaysia Pahang (UMP), 26600 Pekan, Pahang Darul Makmur, \\ Malaysia.
}

\begin{abstract}
Dynamic gameplay, fast-paced and fast-changing gameplay, where angle shooting (top and bottom corner) has the best chance of a good goal, are the main aspects of handball. When it comes to the narrow-angle area, the goalkeeper has trouble blocked the goal. Therefore, this research discusses image processing to investigate the shooting precision performance analysis to detect the ball's accuracy at high speed. In the handball goal, the participants had to complete 50 successful shots at each of the four target locations. Computer vision will then be implemented through a camera to identify the ball, followed by determining the accuracy of the ball position of floating, net tangle and farthest or smallest using object detection as the accuracy marker. The model will be trained using Deep Learning (DL) models of YOLOv2, YOLOv3, and Faster R-CNN and the best precision models of ball detection accuracy were compared. It was found that the best performance of the accuracy of the classifier Faster R-CNN produces 99\% for all ball positions.
\end{abstract}

ARTICLE HISTORY

Received: $5^{\text {th }}$ Nov 2020

Revised: 26 $6^{\text {th }}$ Nov 2020

Accepted: 14 $4^{\text {th }}$ Dec 2020

\section{KEYWORDS}

Handball

Accuracy

High Speed Ball

Deep Learning

Object Detection

\section{INTRODUCTION}

The game of handball is appreciated at varying levels in different parts of the world. Besides, it is hugely popular in France, Hungary, Germany, Poland, Russia and Romania. There are semi-professional leagues of handball in some countries. However, the TV coverage of the sport is only limited to World Championships and other international competitions. This fastball sport required quick analysis in the fast changing circumstances of the game and adapted well to different conditions and needs, creating fun and engaging variations that anyone can play anywhere at any time [1]. Like other team sports, shooting a ball at the goal is the culmination of an offensive phase. Success or failure depends on whether a team attains its ultimate aim, that of scoring a goal. Throwing efficiency is the key to winning or losing matches. It depends mainly on the accuracy and speed of a throw [2]. Nevertheless, the challenging part of this sport is the sizing of the goal post, as the size of the goal post is relatively small, standing at $2 \mathrm{~m}(6.56 \mathrm{ft})$ tall and $3 \mathrm{~m}(9.84 \mathrm{ft})$ wide, compared to football. Thus, shooting accuracy is a crucial skill that needs to be strengthened during shooting training.

A handball goal marker tool for shooting accuracy performance analysis was proposed on this project by realising ball detection. It is due to the existing research in handballs' training and sound experience as a handball athlete [3]. The research proposed a ball shooting system and the best instrument to solve the scoring monitoring system. Unfortunately, the research stated that the analysis showed that the target was not correct as the ball did not exceed its entire circumference.

Therefore, this research was carried out to develop a training tool for the handball sport, mainly for ball detection during shooting. The dataset of different ball positions was collected to train the dataset using deep learning. Instead of using sensors as the ball detection, image processing object detection is proposed in this paper. The remaining part of the paper starts with an elaboration beginning of the related works accordingly. The methodology will be clarified, where systematic steps on developing the training tools will be deliberate in detail. Then, laying out the results as well as associated discussion and lastly, to conclude the paper.

\section{RELATED WORK}

Recent development in sport training applications using image processing has led to renewed interest in machine learning techniques [4]. However, the machine-learning concept's training tools are mostly found for football, athletics, and tennis sports. A training tool was developed, the automated monitors for hitting load for tennis [5]. In this research, the methods were used on 19 competitors that wore an inertial estimation unit (IMU) on their wrist. During the 66 videorecorded instructional courses, the result for all 10-fold cross-validation using a cubic-kernel Support Vector Machine (SVM) was classified into three types of shots with an overall accuracy of 97.4\%. Later in 2020, catch detection for American football using neural network (NN) in machine learning has been established. The method uses a sensor stage 
to get nine degrees of freedom and audio data for both hands in 759 endeavours to get a pass [6]. After pre-processing, the accumulated information was utilised for training the NN to categorise all endeavours, giving an accuracy of $93 \%$.

Besides that, a profound learning network, called TrackNet where it uses a heatmap-based learning network, is prepared to perceive the ball picture from a solitary edge and take in flying status from continuous frames [7]. The result obtains for the TrackNet reach $99.7 \%$ for precision, $97.3 \%$ for recall and $98.5 \%$ for F1-measure.

Moreover, detecting the ball position was used to identify the scoring goal concerning the location of the goalpost in the image [8]. Supervised learning, the SVM model was used for ball detection classification. There were 900 images tested and classified. The goal detection for the visible ball is $98.3 \%$ with a $0.2 \%$ false positive, while detecting the ball during occluded situation the detection rate is $76.2 \%$ with $2.6 \%$ false positive.

Some significant approaches and principles are considered beneficial and provide a lot of assistance for this research after all the possibilities have been collected from the literature. In conclusion, this mixture of transfer learning and machine learning can improve the accuracy performance for ball detection classification.

\section{METHODOLOGY}

\section{Experimental setup}

The research is divided into several levels. The first step is the experimental setup that has to be compatible with image processing, transfer learning, ball and objective detection methods, image processing, and suitable accuracy methods. The second phase of this study was analysing the ball's condition and ensuring that the video angle was correctly taken for the successful ball positions classification. In this phase, the video will be split, and the ball is labelled as 'ball' while the successful goal was grouped according to the classification required, which is the successful goal at the targeted area. The third phase of this study will concentrate on training the dataset with a pre-trained model from different model types with the transfer learning method. Therefore, the step of this project was fast detection ball using the deep learning techniques to get the best accuracy performance on classifying the ball. In developing the training tools, accuracy analysis for the successful goal at the targeted area, the video angle is taken, shooting training and high possibility of successful goal were studied and analyse to obtain ball and goal in the frame. From the video captured during the shooting training, the image was extracted according to the ball's successful goal and visibility.

The data collection that involves the successful goal was carried out at Universiti Malaysia Pahang (UMP) outdoor handball court in Pekan, Pahang. The participants who volunteered for the shooting training are two players from the UMP handball team, female and male, with 11 years and 7 years of experience, respectively. The video was captured using EKEN H9R Action Camera and iPhone X camera in Full HD quality. Figure 1 (a) shows the Action Camera and phone camera placement during the video recorded. Action Camera was located near the 11.5-meter line from the goalpost, while phone cameras are located at the penalty line, which is 7-meters from the goal D-line as in Figure 1 (b). Besides that, the video consist of three type shooting practice: standing, jump, and winger shooting both left and right positions. There are 100 successful goal shots by both players, with 50 each shooting attempt.

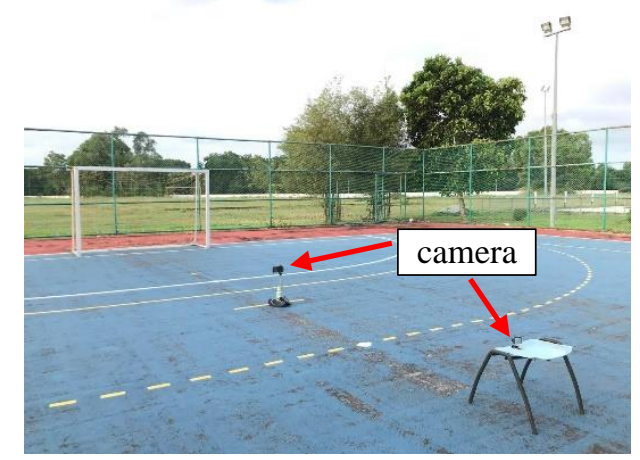

(a)

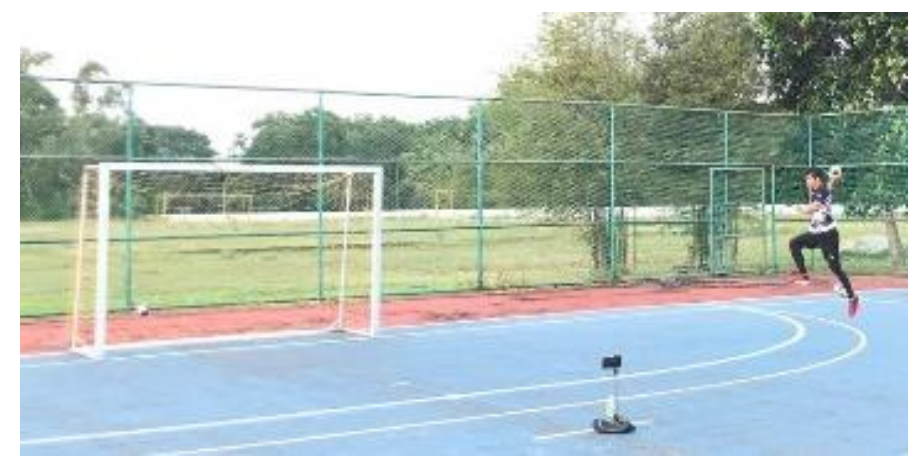

(b)

Figure 1. (a) Shooting video setup (b) shooting angle from outside the D-line

\section{Data Pre-processing}

Pre-processing applies until it is put in the machine learning or deep learning algorithm for all raw data transformation. The positioning of raw data or images has a high risk of producing poor classification results. Therefore, the raw file format that needs to be modified in this project is .xml to .txt because the data will be trained in YOLO and Faster RCNN algorithm. The flow of data pre-processing is shown in Figure 2. 


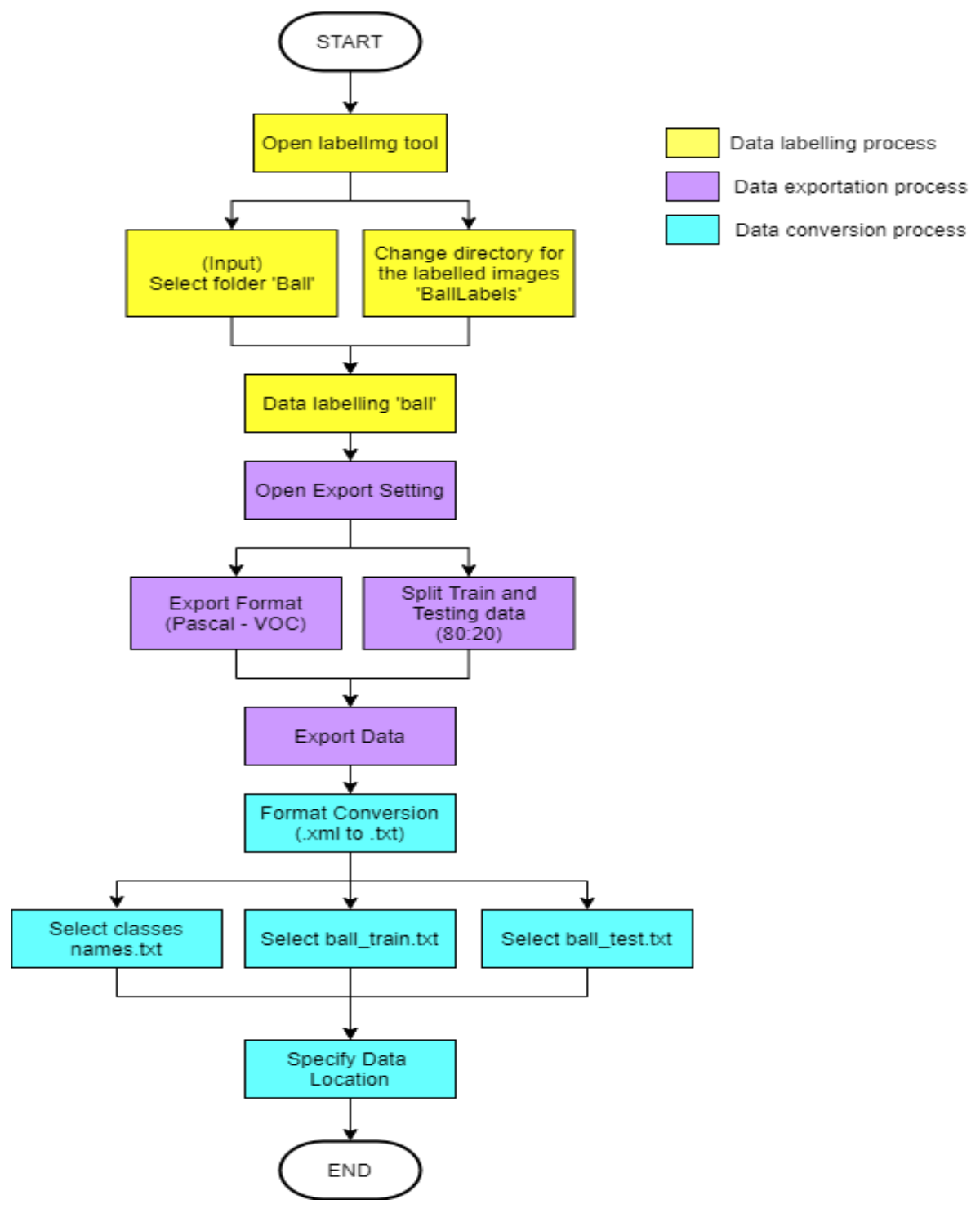

Figure 2. Data Pre-processing flowchart

\section{Data Labelling}

Data labelling terms imply identifying the raw data by marking it to provide meaning. This study refers to the ball, so the deep learning model can correctly learn the data. The bounding box used for data labelling is a rectangular box to denote the object's position where the ball's position is to define in two coordinates, $x$ and $y$-axis as in Figure 3 . To label the data, the labelling tools used are the LabelImg tools. This tool is a Python-based graphical image annotation tool that uses $\mathrm{Qt}$ for its graphical interface. The list or annotation dataset is stored in the Pascal VOC (Visual Object Classes) in XML file, which also supports the YOLO format.

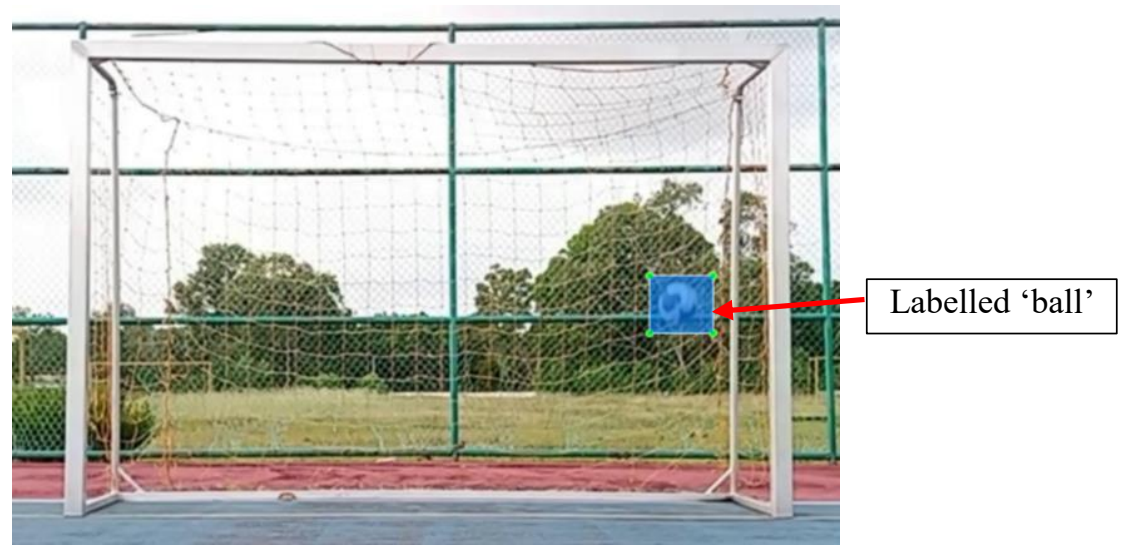

Figure 3. Labelled of the ball's position 


\section{Train and Test Data Split}

In machine learning, training and test data division are essential since this is a method for evaluating the output of a machine learning algorithm. There is a distinction between training and testing data, where training data is implemented in creating a model, while testing data means a collection used to verify the model designed. In addition, the percentage of train data should be more significant than test data for data splitting percentages of train and test datasets. This research dataset, 80:20 ratio, was used to split the dataset for ball dataset, which accumulated to 900 ball images.

\section{Computer Vision Setup}

Computer vision uses machine learning approaches to model image recognition to identify patterns for picture perception [9]. It can help to classify the object according to its classes and detect the object. It is a type of signal processing in which an image is entered, and it is possible to output the function associated with that image. In this project computer, vision will be focussing on ball detection. For ball detection, transfer learning techniques were implemented using a pre-trained model from different object detection algorithms: YOLOv2, YOLOv3 and Faster R-CNN.

\section{Transfer Learning}

Transfer learning is a deep learning technique in which the neural network model is first trained on a problem identical to the problem being resolved. One or more layers of a trained model are then used in a new model trained on the topic of concern. The pre-trained model used is from YOLOv2, YOLOv3, as YOLO's successful object detection was trained to detect 80 different objects, and Faster R-CNN, where its softmax layer has been used to predict the target of the proposed region. An easier understanding of transfer learning is depicted in Figure 4. Model A is the model that has already been trained with multiple objects which are in a large dataset. Then, after model A are fully trained, include model B, which detects specifically ball, therefore during the transfer learning, the training and testing set should be only referred to as class 'ball', as described in Table 1.

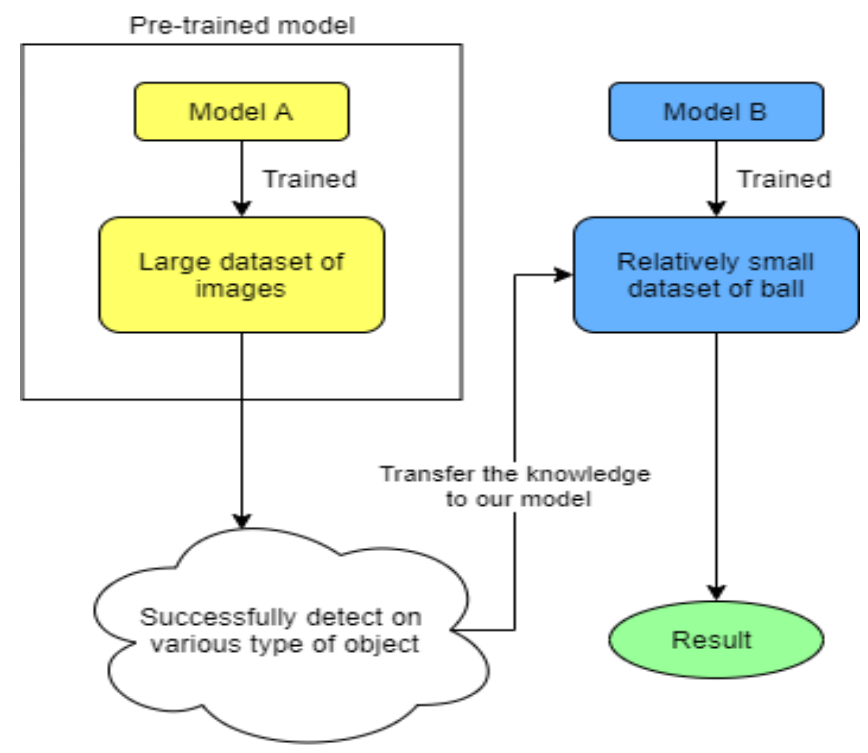

Figure 4. Transfer Learning flowchart

Table 1. Called out 'ball' for training model pseudocode

\begin{tabular}{l}
\hline \multicolumn{4}{l}{ Algorithm: called out ball } \\
\hline 1: $\quad$ runMode $=$ "train" \\
2: $\quad$ classes $=[$ "Ball"] \\
\hline
\end{tabular}

\section{RESULTS AND DISCUSSION}

\section{Condition and position accuracy performance}

The accuracy performance of the ball detection is selected according to its condition and position. There are three types of position and condition: floating ball, ball condition when tangle with net and the farthest and small ball position refer to Figure 5. From the average accuracy, the result shows in Figure 6, and the result is taken for floating, net tangle and farthest position of the ball, YOLOv3 improve to $9 \%, 20 \%$ and $22 \%$, respectively from the YOLOv3 model. However, 
Faster R-CNN has the best accuracy performance with steady $99 \%$ accuracy in detecting the ball compare to YOLO in all conditions.

(a)

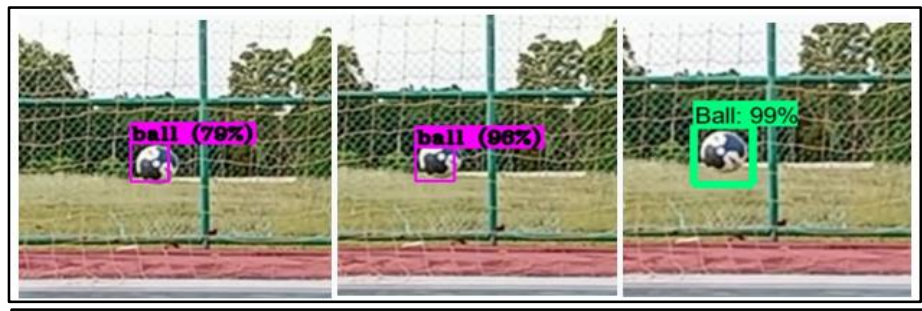

(b)
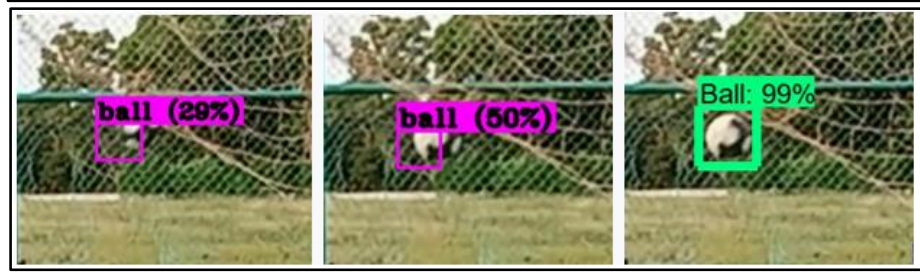

(c)

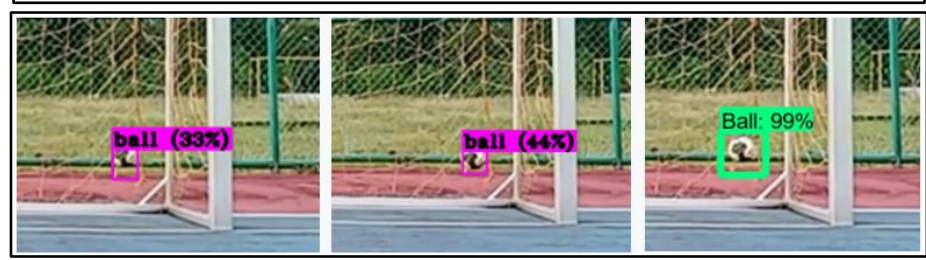

Figure 5. (a) Frame 181 of floating condition (b) Frame 21 of ball tangle on the goal net (c) Frame 576 of farthest and small condition ball

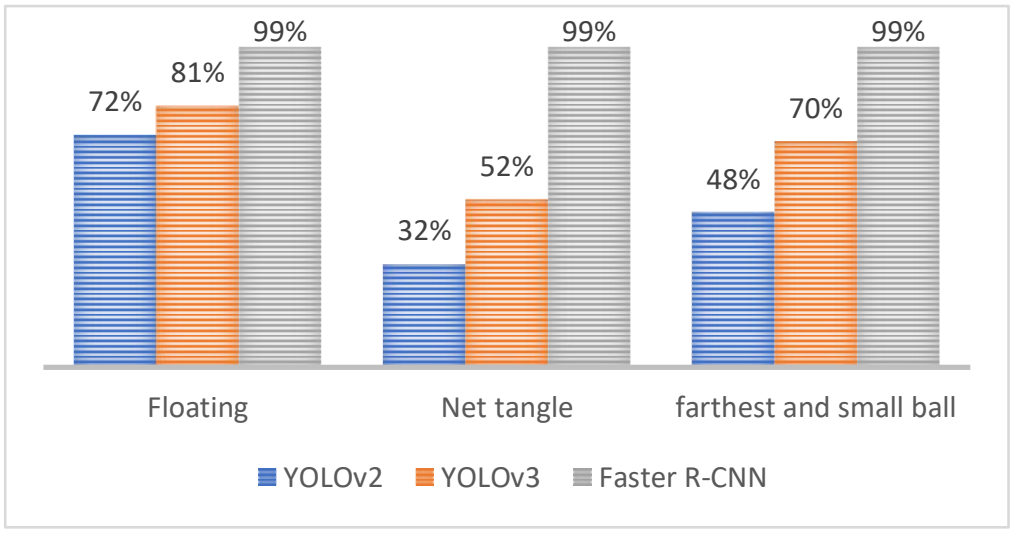

Figure 6. Average accuracy comparison in different conditions and position

\section{Discussion}

As illustrated in Figure 5, the prediction bounding box for the detection was accurately within the size of the actual bounding box of the ball for floating, net tangle, and farthest and small ball. It shows that the Faster R-CNN was adequately trained to detect the ball compared to the other YOLO versions accurately. The Faster R-CNN model shows the best accuracy performance in detecting the high-speed ball with an average accuracy of 99\%. Even though some drawbacks, such as ghosting detection, where the model detects empty areas or other inanimate objects as the ball, it manages to detect the ball efficiently.

\section{CONCLUSION}

In conclusion, the objective of this project in detecting the ball is successful by using the Faster R-CNN model. The model gives $99 \%$ accuracy in detecting the object in all frames. Even though the ball was located at different locations, either further away or floating, the accuracy detection is between $97 \%$ to $100 \%$, albeit the high-speed movement. The current research findings recommend to focus on the real-time implementation of the developed machine learning and increase the training model so that the machine can recognise and have a better accuracy 


\section{ACKNOWLEDGEMENT}

The authors would like to thank the Innovative Manufacturing, Mechatronics and Sports Laboratory (iMAMS Lab) and Faculty of Manufacturing and Mechatronic Engineering Technology of Universiti Malaysia Pahang for cooperating with this research.

\section{REFERENCES}

[1] C. Manchado et al., "High-Performance Handball Player's Time-Motion Analysis by Playing Positions," Int. J. Environ. Res. Public Heal. 2020, Vol. 17, Page 6768, vol. 17, no. 18, p. 6768, Sep. 2020.

[2] J. C. Zapardiel Cortés, C. Ferragut Fiol, C. Manchado, J. A. Abraldes Valeiras, and H. Vila Suárez, "Difference of the speed of handball throwing during the competition in relation to efficiency: Analysis between the first and the second half," Jan. 2018.

[3] S. K. Albzeirat, A. Elmetwaly, M. K. Albzeirat, and N. Z. Noriman, "Developing a Smart Monitoring System for Refereeing Goal Setting in Football Developing a Smart Monitoring System for Refereeing Goal Setting in Football Matches," no. April, 2020.

[4] M. N. A. Shapiee, M. A. R. Ibrahim, M. A. M. Razman, M. A. Abdullah, R. M. Musa, and A. P. P. A. Majeed, "The Classification of Skateboarding Tricks by Means of the Integration of Transfer Learning and Machine Learning Models," Lect. Notes Electr. Eng., vol. 678, pp. 219-226, 2020.

[5] D. Whiteside, O. Cant, M. Connolly, and M. Reid, "Monitoring hitting load in tennis using inertial sensors and machine learning," Int. J. Sports Physiol. Perform., vol. 12, no. 9, pp. 1212-1217, Oct. 2017.

[6] B. Hollaus, S. Stabinger, A. Mehrle, and C. Raschner, "Using wearable sensors and a convolutional neural network for catch detection in American football," Sensors (Switzerland), vol. 20, no. 23, pp. 1-16, 2020.

[7] Y. C. Huang, I. N. Liao, C. H. Chen, T. U. Ik, and W. C. Peng, "TrackNet: A deep learning network for tracking high-speed and tiny objects in sports applications," arXiv, 2019.

[8] N. Ancona, G. Cicirelli, A. Branca, and A. Distante, "Goal detection in football by using support vector machines for classification," Proc. Int. Jt. Conf. Neural Networks, vol. 1, pp. 611-616, 2001.

[9] R. Sagar, "What Is The Difference Between Computer Vision And Image Processing," Analytics India Magazine, 26-Dec-2018. [Online]. Available: https://analyticsindiamag.com/what-is-the-difference-between-computervision-and-image-processing/. 